\title{
Praseodymium barium molybdate-its growth and structural characterization
}

\author{
JAYAKUMARI ISAC, M A ITTYACHEN and K S RAJU*' \\ School of Pure and Applied Physics, Mahatma Gandhi University, Kottayam 686031, India \\ ${ }^{\dagger}$ Department of Crystallography and Biophysics, University of Madras, Guindy Campus, \\ Madras 600025 , India \\ MS received 24 November 1994; revised 19 October 1995

\begin{abstract}
Praseodymium barium molybdate (PBM) single crystals grown by gel method, reveal multiple and isolated octahedral bipyramidal crystals as well as spherulites of PBM at different depths from the surface inside the gel. Oscillation X-ray diffraction pattern of the the presence of molybdate $\left(\mathrm{MoO}_{4}\right)^{2-}$ ions in $\mathrm{PBM}$, while the thermogravimetric analysis shows that PBM loses molybdenum oxide component around $420^{\circ} \mathrm{C}$. Energy dispersive $\mathrm{X}$-ray analysis establishes the presence of $\mathrm{Pr}, \mathrm{Ba}$ and $\mathrm{Mo}$ in the sample. X-ray photoelectron spectroscopic studies of PBM establish the presence of $\mathrm{Pr}, \mathrm{Ba}$ and $\mathrm{Mo}$ in their respective oxide states. An empirical structure has been proposed on the basis of these findings. The implications are discussed.
\end{abstract} \\ sample reveals that PBM is single crystalline in nature. Infrared absorption spectrum confirms
}

Keywords. Praseodymium barium molybdate; gel growth; characterization; XRD; IR; TGA; EDAX; XPS.

\section{Introduction}

The rare earth molybdates are of great interest because of their ferroelectric and ferroelastic properties which in turn, find applications in electro and acousto optical devices (Barkley et al 1971, 1972; Sapriel and Vacher 1977). Barium molybdate tetragonal bipyramidal crystals are grown by the precipitation of alkaline earth metal molybdate powders from the neutral aqueous solutions (Pacter 1977). Using the Czochralski crystal growth technique, rare-earth molybdates $(R=N d, S m, P r, E u, T b$ and Dy) are grown at elevated temperatures (Brixner 1973). These crystals owing to thermal stresses are found to be defective. To overcome these defects, methods using crystal growth at room temperatures are preferred. It is well known that for the growth of inorganic crystals, which are sparingly soluble, gel technique pioneered by Henisch et al (1965) and Henisch (1970) is found to be the most suitable. Although there is limitation in the sizes of the gel grown crystals, this technique has been fully exploited for investigating the morphologies of calcium sulphate dihydrate as interpenetration twinning (Raju 1983a), dendritic (Raju 1983b), besides herring-bone and hour glass structures (Jayakumar and Raju 1983), on changing the growth conditions like pH of the gel, different reactants and their concentrations, besides, varying additives to the crystal growing gel medium. Crystals of rare earth mixed oxalates and molybdates grown by this method at room temperatures are characterized by employing several techniques (Raju et al 1994; Sushma Bhat et al 1995). The authors report here the work carried out on the gel grown mixed molybdate crystals of barium and praseodymium,

\footnotetext{
*For correspondence
} 


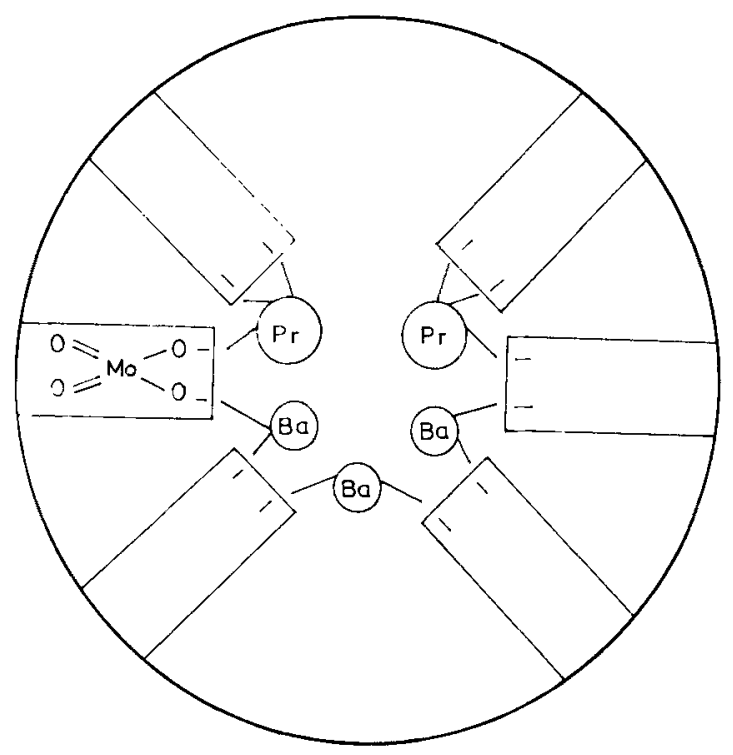

Figure 1. The proposed empirical structure of PBM.

besides, their characterization employing XRD, IR, TGA, EDAX, and XPS studies, which throws light in understanding the kinetics of chemical reactions, the qualitative and quantitative analyses of heavy elements present in the mixed molybdates, and the presence of their chemical states. On the basis of these results the empirical structure of PBM sample has been proposed and discussed.

\section{Experimental observations and results}

\subsection{Growth of PBM in gels}

Aqueous solution of sodium meta silicate (specific gravity $1.04 \mathrm{~g} \mathrm{~cm}^{-3}$ ) is adjusted to the desired $\mathrm{pH}$ by using analar grade nitric acid and used as the reacting medium. A mixture of aqueous solutions of praseodymium nitrate and barium nitrate with nitric acid is used as the outer reactant above the gel at room temperature. The outer reactant diffuses into the set gel impregnated with ammonium molybdate and the following reaction takes place producing brown colloidal precipitate of mixed molybdates of praseodymium and barium $\left(\mathrm{Pr}_{2} \mathrm{Ba}_{3}\left(\mathrm{MoO}_{4}\right)_{6}\right)$ and ammonium nitrate.

$$
\begin{gathered}
2 \mathrm{Pr}\left(\mathrm{NO}_{3}\right)_{3}^{-} \\
+ \\
3 \mathrm{Ba}\left(\mathrm{NO}_{3}\right)_{2-}
\end{gathered} \mid+6\left(\mathrm{NH}_{4}\right)_{2} \mathrm{MoO}_{4} \rightarrow \mathrm{Pr}_{2} \mathrm{Ba}_{3}\left(\mathrm{MoO}_{4}\right)_{6}+12 \mathrm{NH}_{4} \mathrm{NO}_{3} .
$$

A thick layer of the precipitate is formed just below the gel interface. The nucleation from the precipitate resulting in crystallization has been observed with the formation of large number of tiny multiple crystals (figure 2) and spherulites (see figure 4) underneath it, while well defined isolated octahedral bipyramidal crystals (figure 3) of relatively bigger sizes appear (average size after four weeks: $3 \times 2 \times 1 \mathrm{~mm}^{3}$ ) at the 


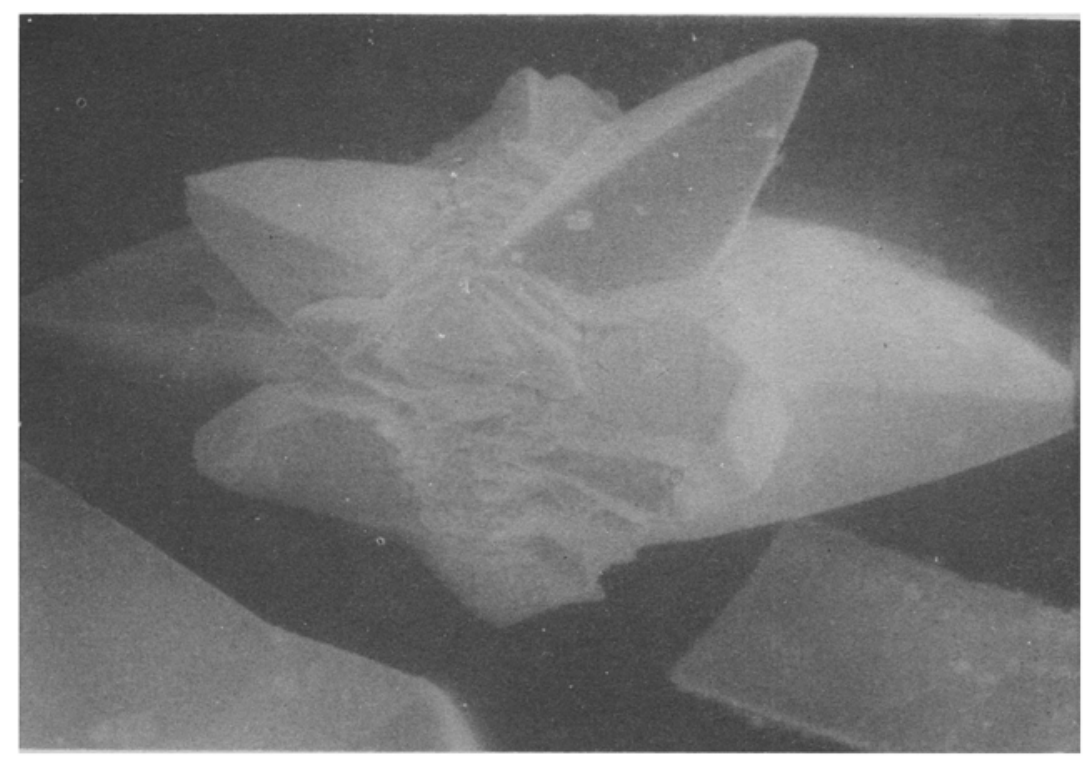

Figure 2. SEM picture of a multiple crystal of PBM $(\times 320)$.

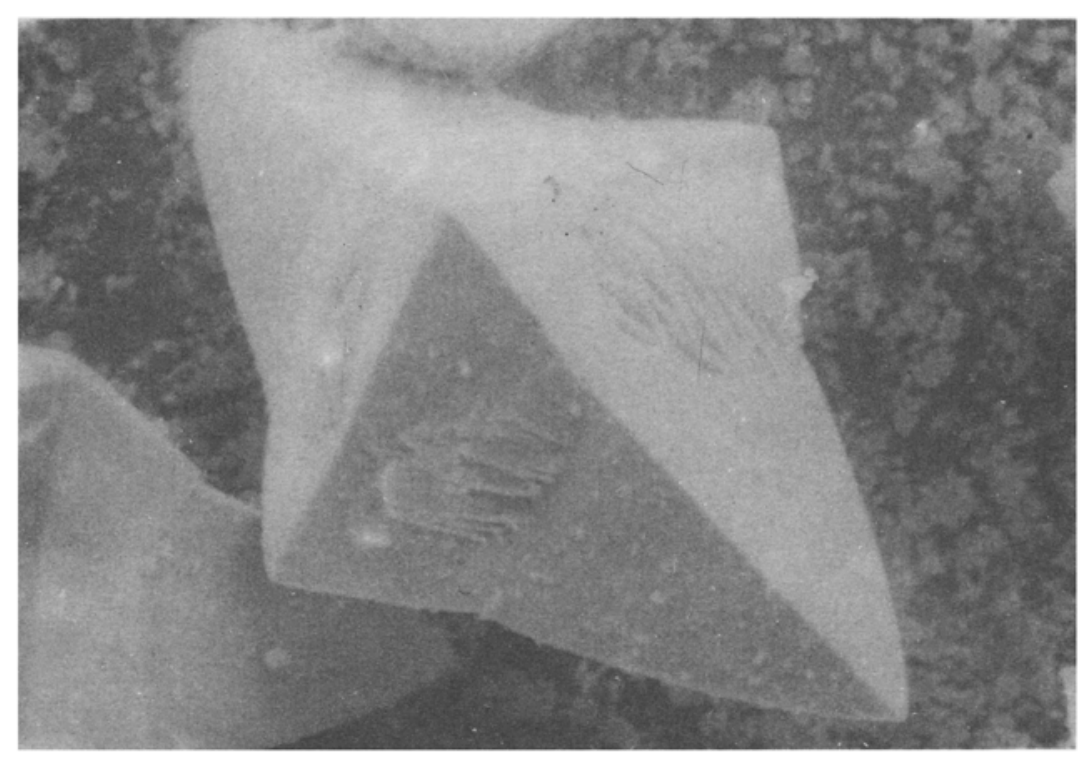

Figure 3. SEM picture showing an octahedral bipyramidal crystal of PBM $(\times 380)$.

greater depths inside the gel. The formation of spherulites in the zone very close to the lower surface layer of the precipitate (also the region of multiple crystals) is explained. The free colloidal particles of the precipitate occupy a larger volume, and after the appearance of an active nucleation site, the reacted ions tend to attain a smaller volume around it, thereby condensing into a spherulitic crystal. The spherulitic morphology in 


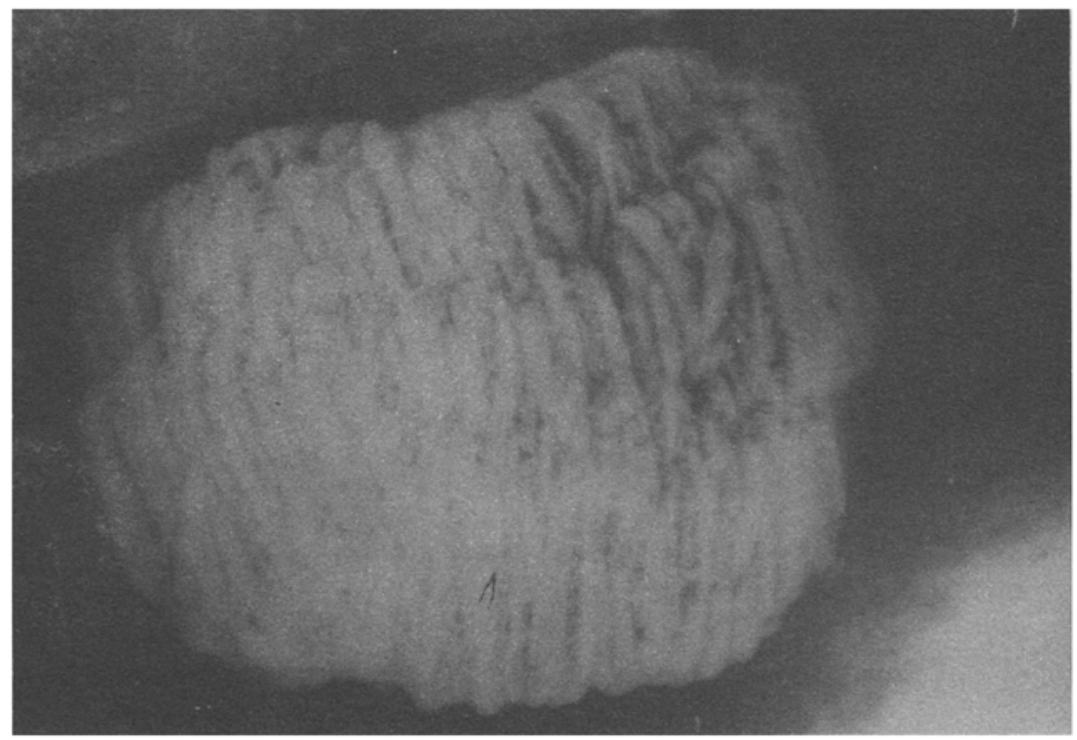

Figure 4. SEM picture of a spherulite of PBM $(\times 1150)$.

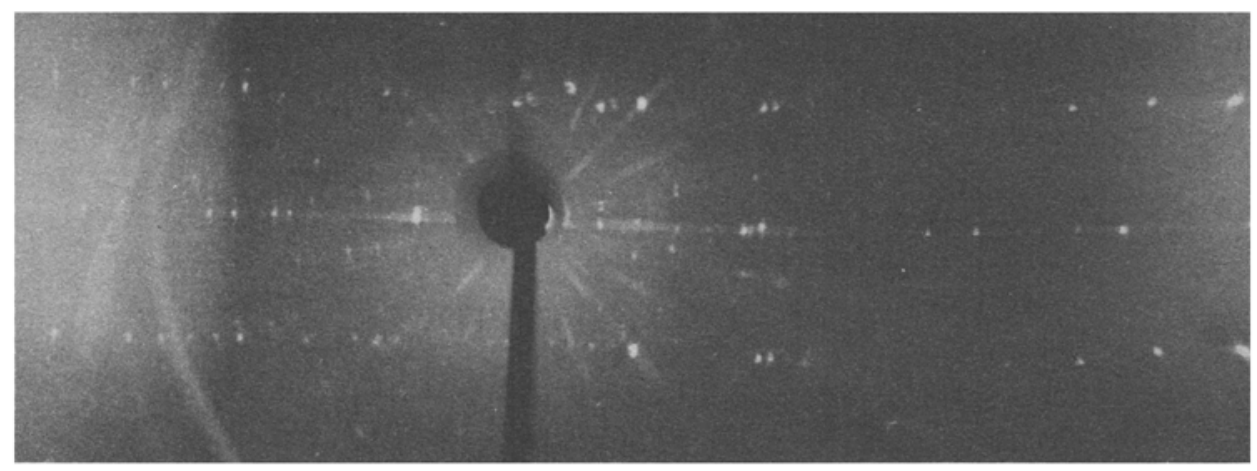

Figure 5. Oscillation XRD photograph of PBM.

the case of gel grown rare earth tartrates (Anima Jain et al 1991) has been explained as due to the agglomeration of microcrystallites in the spherical shape.

The optimum conditions for the growth of good single crystals are found to be at $\mathrm{pH}$ of 6 of the gel with 0.5 molarity of each of the nitrates in $(1: 1)$ by volume in the outer reactant.

The proposed empirical structure of praseodymium barium molybdate is given in figure 1.

\subsection{Characterization of $P B M$}

2.2a XRD studies: The oscillation XRD pattern of PBM sample (figure 5) is taken with a Unicam camera with $\mathrm{CuK}_{\alpha}$ radiation $(\lambda=1.5418 \mathrm{~A})$. The revelation of well 


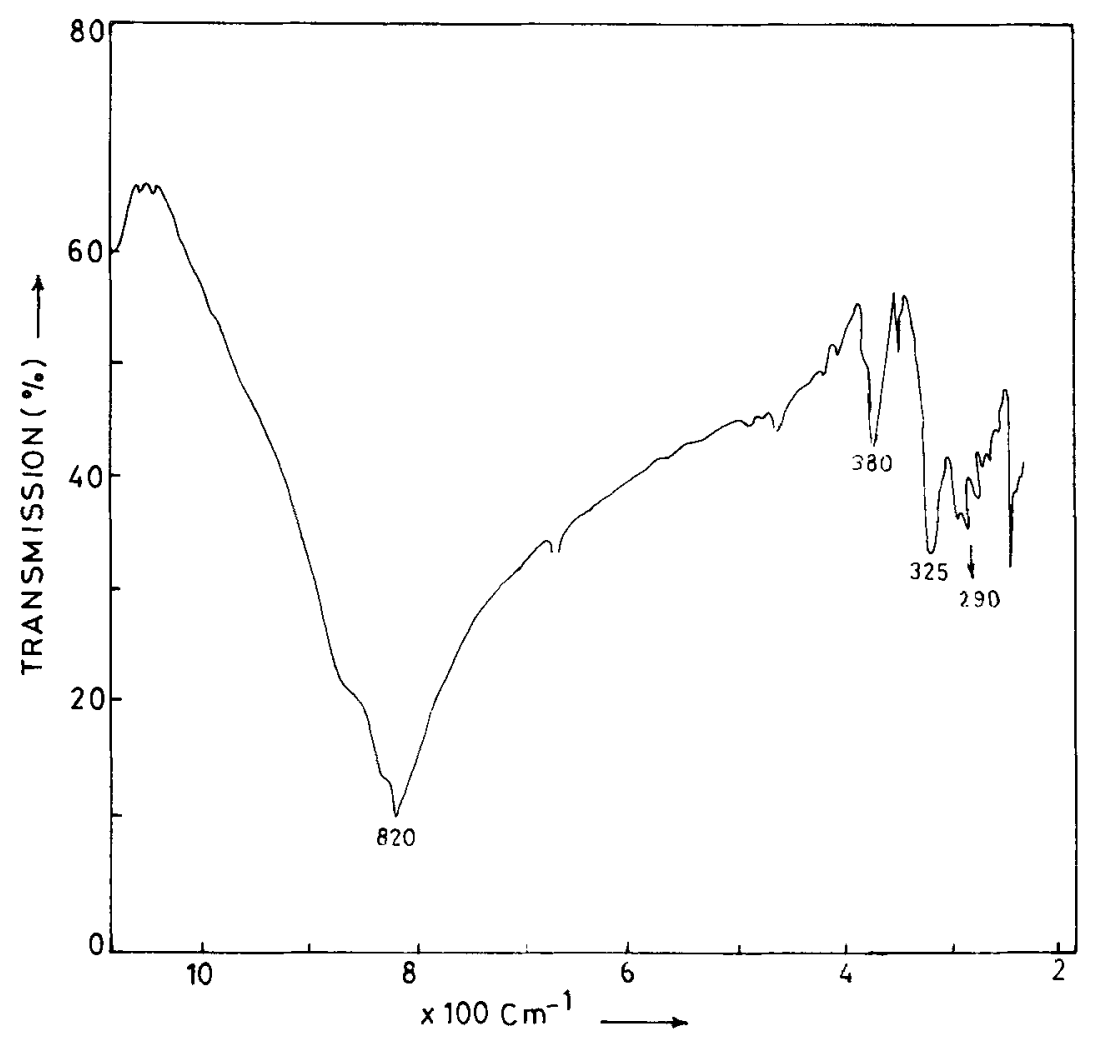

Figure 6. IR absorption spectrum of PBM.

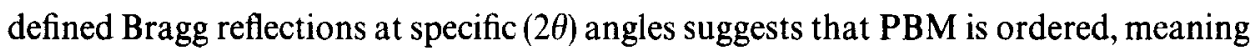
single crystalline in nature.

2.2b IR absorption studies: The IR absorption spectrum of PBM sample (figure 6) taken in $\mathrm{KBr}$ matrix, is recorded in IR-PERKIN ELMER model 530, England.

That the PBM sample has molybdate $\left(\mathrm{MoO}_{4}\right)^{2-}$ ions is supported by the revelation of well pronounced broad intense peak at $820 \mathrm{~cm}^{-1}$ relating to the symmetric or antisymmetric stretching modes of vibrations (Busy and Keller 1964), while peak at $380 \mathrm{~cm}^{-1}$ relates to antisymmetric bending vibrations and the peaks at 325 and $290 \mathrm{~cm}^{-1}$ relate to symmetric bending vibrations (Clark and Doyle 1966). It may be mentioned that these assignments for molybdate $\left(\mathrm{MoO}_{4}\right)^{2-}$ agree quite well with the literature findings for samarium barium molybdate (Jayakumari Isac and Ittyachen 1992).

2.2c TGA of PBM: The thermogravimetric plot of PBM crystal was taken using Delta series TGA 7 at the scanning of $20^{\circ} \mathrm{C} / \mathrm{min}$ (figure 7 ). This shows that the PBM crystal undergoes a remarkable loss of weight around $420^{\circ} \mathrm{C}$ and this may be attributed to the preferential vaporization of the molybdenum oxide component, according to the following equation

$$
\mathrm{Pr}_{2} \mathrm{Ba}_{3}\left(\mathrm{MoO}_{4}\right)_{6} \rightarrow \mathrm{Pr}_{2} \mathrm{O}_{3}+3 \mathrm{BaO}+6 \mathrm{MoO}_{3}(g),
$$

and the residue at $870^{\circ} \mathrm{C}$ is found to be $2 \cdot 885 \%$ only. 


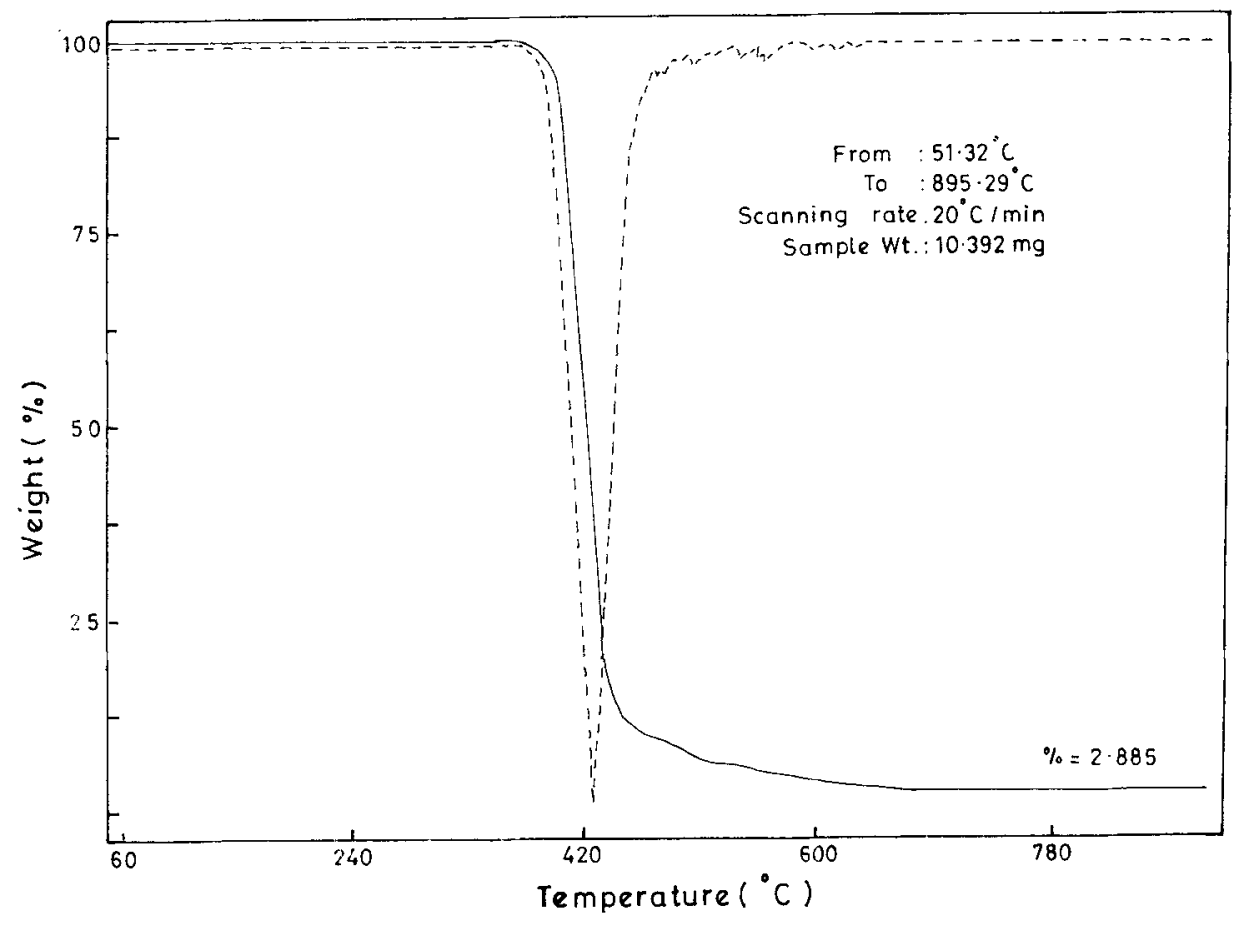

Figure 7. TGA plot of PBM.

2.2d EDAX of PBM: EDAX work has been taken up in order to establish the elemental incorporation like $\mathrm{Pr}, \mathrm{Ba}$ and Mo in the PBM crystals. The surfaces of PBM crystals mounted onto aluminium stubs are gold coated to make them electrically conducting and are examined in the EDAX analyser No. 711, an accessory to the scanning electron microscope, Philip's SEM model 501. The EDAX patterns of PBM sample are shown as continuous lines in figures $8 \mathrm{a}, \mathrm{b}$.

The two peaks positioned at 5.03 and $5.5 \mathrm{keV}$ relate to $L_{\alpha}$ and $L_{\beta}$ energies of praseodymium, while $L_{\alpha}$ and $L_{\beta}$ energies relating to barium are represented by peaks positioned at 4.5 and $4.8 \mathrm{keV}$ respectively (figure $8 \mathrm{a}$ ). The peak at $2.3 \mathrm{keV}$ (figure $8 \mathrm{~b}$ ) relates to $\operatorname{Mo}\left(L_{\alpha}\right)$, whereas a shoulder at $2.4 \mathrm{keV}$ corresponds to $\operatorname{Mo}\left(L_{\beta}\right)$ and hence is broad. It is interesting to note that to the left of the broad Mo peak, a weak hump at $1.75 \mathrm{keV}$ corresponds to silicon $\left(\mathrm{K}_{\alpha}\right)$. This gives a strong clue that PBM grown in gel medium has 'gel' inclusions in them, as 'gel' in the present case is sodium meta silicate. The intensity of $\mathrm{Si}\left(\mathrm{K}_{\alpha}\right)$ peak reduces considerably for PBM crystals grown at greater depths (isolated octahedral bipyramidal crystals), meaning lesser gel inclusion as the depth increases. It may be mentioned that the kinetic energy values reported for $\operatorname{Pr}\left(L_{\alpha, \beta}\right), \operatorname{Ba}\left(L_{\alpha, \beta}\right), \operatorname{Mo}\left(L_{\alpha, \beta}\right)$ and $\operatorname{Si}\left(\mathrm{K}_{\alpha}\right)$ in the present EDAX investigations are in excellent agreement with the EDAX library.

2.2e XPS studies: XPS studies are undertaken in order to further establish the constituents incorporated in PBM sample besides their chemical states.

PBM is finely powdered and a pellet of $1 \mathrm{~cm}$ diameter and $1 \mathrm{~mm}$ thickness is formed using a hydraulic press by applying a pressure of $1 \cdot 1$ tons per $\mathrm{cm}^{2}$. XPS spectra of PBM 

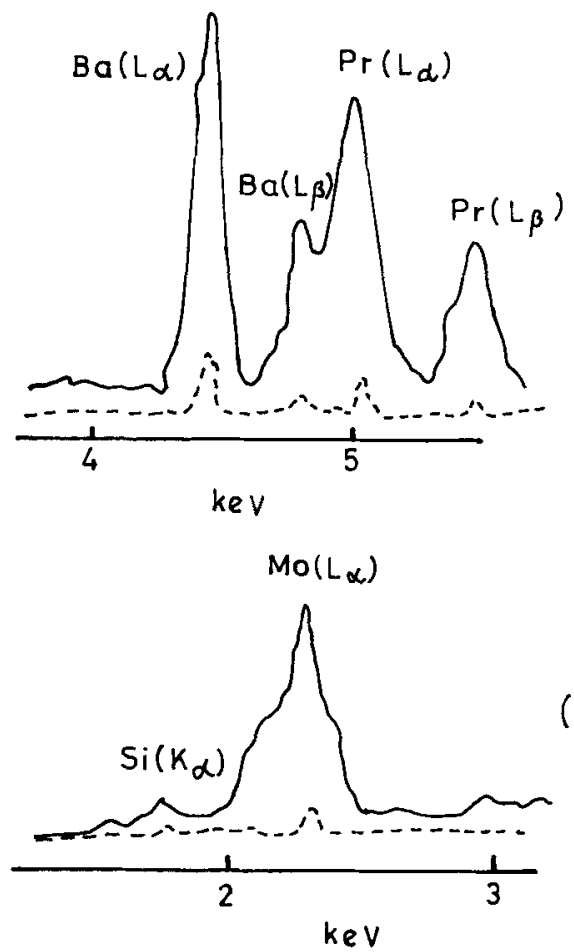

(a)

(b)

Figure 8. EDAX patterns of $\mathrm{PBM}$ (a) regions showing Pr and $\mathrm{Ba}$ and (b) regions showing Mo and $\mathrm{Si}$ (solid line) and of TGA residual PBM at $800^{\circ} \mathrm{C}$ (dashed line).

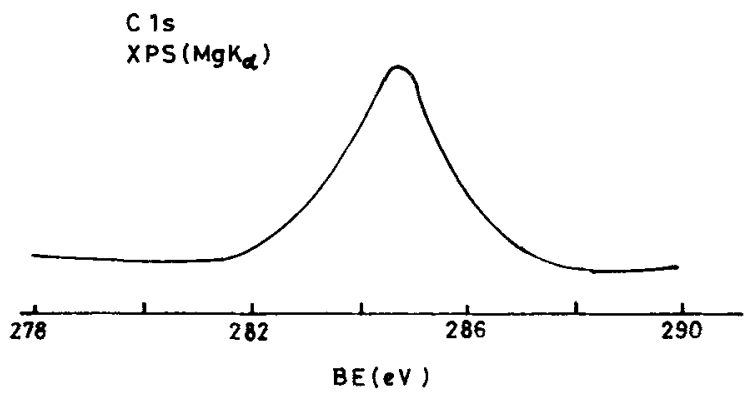

Figure 9. XPS peak of $C 1 s$ of PBM.

pellet are taken in VG scientific ESCALAB MK II, by irradiating the sample with the monochromatic soft $\mathrm{X}$-rays $\left(\mathrm{Mg} \mathrm{K}_{\alpha}\right.$; photon energy $\left.1253.6 \mathrm{eV}\right)$. The constant analyser energy is $50 \mathrm{eV}$ and the resolution of the system is about $0.7 \mathrm{eV}$. XPS peak of $C 1 \mathrm{~s}$ (figure 9) denotes the binding energy of $284.6 \mathrm{eV}$ which agrees quite well with the reported value in the literature (Wagner et al 1978) and hence the binding energy scale (X-axis) is calibrated. The presence of carbon in the present investigations is attributed to the adventitious hydrocarbon nearly always present from the laboratory environment or from a glove box.

Figure 10 shows the XPS peak relating to $\operatorname{Pr}\left(3 d_{5 / 2}\right)$ with the binding energy centred at $930 \mathrm{eV}$, while figure 11 relates to the XPS peaks of $\mathrm{Ba}\left(3 d_{5 / 2}-3 d_{3 / 2}\right)$ with the binding 


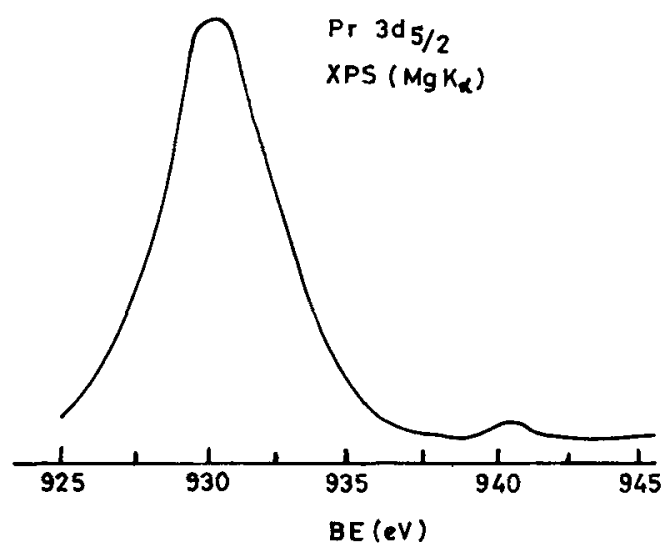

Figure 10. XPS peak of $\operatorname{Pr}\left(3 d_{s / 2}\right)$ of $P B M$.

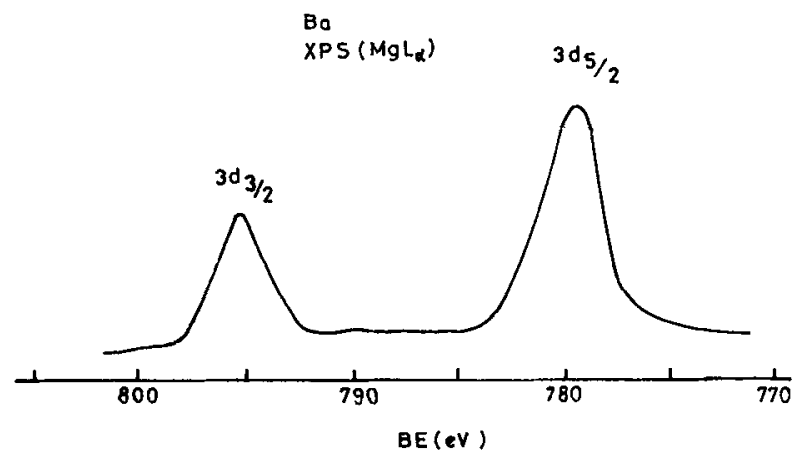

Figure 11. XPS peaks of $\mathrm{Ba}\left(3 d_{5 / 2}-3 d_{3 / 2}\right)$ of $\mathrm{PBM}$.

energies around 780 and $795 \mathrm{eV}$, with the separation of $15.4 \mathrm{eV}$ and figure 12 shows the XPS peaks of $\mathrm{Mo}\left(3 d_{5 / 2}-3 d_{3 / 2}\right)$ having the binding energies around 232.5 and $235.7 \mathrm{eV}$, with the separation of $3 \cdot 2 \mathrm{eV}$ respectively. The binding energy values relating to $\operatorname{Pr}\left(3 d_{5 / 2}\right)$ and $\mathrm{Ba}\left(3 d_{5 / 2}-3 d_{3 / 2}\right)$ and $\operatorname{Mo}\left(3 d_{5 / 2}-3 d_{3 / 2}\right)$ along with their separations (figures 10-12) agree quite well with the literature values (Wagner et al 1978) corresponding to their oxide states $\left(\operatorname{Pr}_{2} \mathrm{O}_{3}, \mathrm{BaO}\right.$ and $\left.\mathrm{MoO}_{3}\right)$.

Thus, it is well established that XPS, though a surface analysis, is a powerful technique in identifying the constituents present in the sample and also their respective chemical states.

\section{Discussion}

That the PBM grown by gel is crystalline in nature is evident by the revelation of multiple crystals and spherulites nearer to the thick precipitate layer at the gel interface within, while octahedral bipyramidal isolated crystals are observed at the greater depths in the gel (figures 2-4). The faster rate of diffusion nearer to the gel interface gives rise to multiple crystals, while slower rate of diffusion at the greater depths gives 


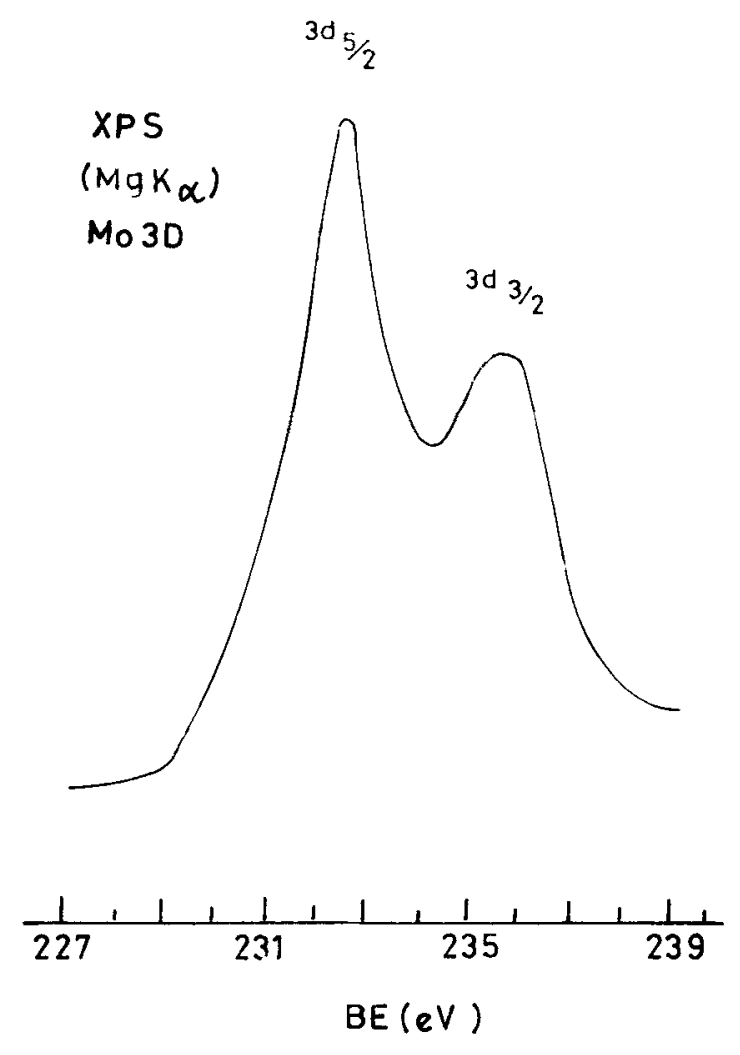

Figure 12. XPS peaks of $\mathrm{Mo}\left(3 d_{5 / 2}-3 d_{3 / 2}\right)$ of PBM.

rise to isolated octahedral bipyramidal crystals with well defined edges and corners. This reasoning explains more 'gel inclusion' in multiple and spherulitic crystals (figure $8 \mathrm{~b}$ ) than in isolated crystals formed by slower diffusion rates. The single crystalline nature of the PBM sample is further ascertained by its oscillation photograph of X-ray diffraction (figure 5). The identical XRD patterns of the powdered spherulites and the powdered multiple and octahedral bipyramidal XRD patterns of isolated crystals strongly suggest that the spherulitic crystals are formed due to the agglomerations of microcrystallites (Anima Jain et al 1991).

The IR absorption spectrum of PBM (figure 6) establishes the presence of molybdate complex $\left(\mathrm{MoO}_{4}\right)^{2-}$ in the sample. TGA of PBM reveals that the crystals lose molybdenum oxide component around $420^{\circ} \mathrm{C}$, with $100 \%$ weight of $\mathrm{PBM}$ till $330^{\circ} \mathrm{C}$ reducing to $2.885 \%$ by weight beyond $800^{\circ} \mathrm{C}$. The EDAX studies confirm the incorporation of $\mathrm{Pr}$, $\mathrm{Ba}$ and Mo in PBM sample. The heights and or areas (Riggs and Parker 1975) of $L_{\alpha}$ peaks of $\mathrm{Pr}$ and $\mathrm{Ba}$ give the clue that both may have approximately the same affinity to their molybdate complex formation. The EDAX pattern of the PBM residue of TGA experiment (dotted lines in figures $8 \mathrm{a}$ and b) around $800^{\circ} \mathrm{C}$ confirms the conjecture that the loss of weight of $\mathrm{PBM}$ around $420^{\circ} \mathrm{C}$ is due to the preferential vaporization of the molybdenum oxide component, by the revelation of a weak hump at $2.4 \mathrm{keV}$ relating to $\operatorname{Mo}\left(L_{\alpha}\right)$ [dotted line in figure 8b]. The heights and/or areas of $\operatorname{Pr}\left(L_{\alpha}\right.$ and $\left.L_{\beta}\right)$ and $\mathrm{Ba}\left(L_{\alpha}\right.$ and $L_{\beta}$ ) peaks (dotted line in figure 8a), strongly suggests that the residue constitutes mainly of $\mathrm{Pr}_{2} \mathrm{O}_{3}$ and $\mathrm{BaO}$, with the traces of $\mathrm{MoO}_{3}$ (dotted line in figure 8b). 
It is observed that the intensities of $\mathrm{Pr}$ and $\mathrm{Ba}$ in TGA residue (dotted line in figure 8a) have lesser values than expected, which may be attributed due to the 'adsorption' of $\mathrm{MoO}_{3}$ in layers of atomic thickness, on $\mathrm{Pr}_{2} \mathrm{O}_{3}$ and $\mathrm{BaO}$ residue, thereby reducing the intensities of Pr and $\mathrm{Ba}$ peaks in EDAX and revealing Mo in traces, even after the heating and cooling in TGA experiment.

The XPS results support well that $\mathrm{Pr}, \mathrm{Ba}$ and $\mathrm{Mo}$ are in their oxide states (figures 10-12), getting linked to $\mathrm{O}^{-}$of $\left(\mathrm{MoO}_{4}\right)^{2-}$. These results are in excellent agreement with the proposed empirical formula for PBM (figure 1).

\section{Conclusion}

In conclusion it can be said that the gel grown PBM sample reported in the present work (i) is ordered and crystalline as evidenced by the formation of well defined multiple crystals, spherulites and octahedral bipyramidal isolated crystals (figures 2-4), (ii) is single crystalline in nature (XRD), (iii) accounts for the presence of molybdato complex by the revelation of their characteristic peaks (IR), (iv) loses molybdenum oxide component around $420^{\circ} \mathrm{C}$ (TGA), (v) has $\mathrm{Pr}$, Ba and Mo established qualitatively and to some extent quantitatively, with $\mathrm{Si}$ trapped in multiple and spherulitic crystals (EDAX) and (vi) is having $\mathrm{Pr}, \mathrm{Ba}$ and $\mathrm{Mo}$ in their oxide states getting linked to $\mathrm{O}^{-}$of molybdate groups (XPS) (figure 1).

\section{Acknowledgement}

Authors thank the Chairman, Materials Research Centre, Indian Institute of Science, Bangalore, for XRD and IR, Prof. V S Raghunathan, Head, Metallurgy Division; IGCAR, Kalpakkam, for EDAX and Regional Sophisticated Instrumentation Centre, Indian Institute of Technology, Madras, for XPS facilities.

\section{References}

Anima Jain, Razdan A K and Kotru P N 1991 Mater. Sci. \& Engg. B8 129

Barkley J R, Brixner L H and Hogan E M 1971 IEEE symp. on the applications of ferroelectrics (New York: York Town Heights)

Barkley J R, Brixner L H, Hogan E M and Waring R K 1972 J. Ferroelectrics 3191

Brixner L H 1973 J. Cryst. Growth 18297

Busy R H and Keller O L Jr 1964 J. Chem. Phys. 41215

Clark Q M and Doyle W P 1966 Spectrochim. Acta 221441

Henisch H K 1970 Crystal growth in gels (Pennsylvania: Pennsylvania University Press)

Henisch H K, Dennis J and Hanoka J I 1965 J. Phys. Chem. Solids 26493

Jayakumar D and Raju K S 1983 Bull. Mater. Sci. 5399

Jayakumari Isac and Ittyachen M A 1992 Bull. Mater. Sci. 15349

Pacter A 1977 Krist. Tech. 12729

Raju K S 1983a J. Mater. Sci. Letts. 2705

Raju K S 1983 b Cryst. Res. \& Technol. 181277

Raju K S, Krishna K N, Jayakumari Isac and Ittyachen M A 1994 Bull. Mater. Sci. 171447

Riggs W M and Parker M J 1975 in Methods of surface analysis (ed.) A W Czanderna (Amsterdam: Elsevier Scientific Pub. Co.) Chap. 4 p. 108

Sapriel J and Vacher R 1977 J. Appl. Phys. 481191

Sushma Bhat, Kotru P N and Raju K S 1995 Curr. Sci. 69607

Wagner C D, Riggs W M, Davis L E, Moulder J F and Mullenberg J E (ed.) 1978 Handbook of X-ray photoelectron spectroscopy (Minnesota, USA: Perkin Elmer, physical electronics division) 